\title{
Predictors of Metastasis and Outcome Following True Negative Sentinel Node Biopsy
}

\author{
Johanna S Palve ${ }^{1 *}$, Tiina H Luukkaala², Minna T Kääriäinen ${ }^{1}$ \\ ${ }^{1}$ Tampere University Hospital, Departments of Plastic Surgery, Tampere, Finland \\ ${ }^{2}$ Research, development and innovation Center, Tampere University Hospital and Health Sciences, Faculty of Social \\ sciences, Tampere University, Tampere, Finland
}

\section{*Corresponding Author \\ Johanna S Palve, Tampere \\ University Hospital, Department of \\ Plastic Surgery, Teiskontie 35, \\ 33521 Tampere, Finland, Tel: \\ +358331167506; \\ E-mail: \\ johanna.palve@pshp.fi}

Received: 20 August 2019;

Accepted: 04 September 2019;

Published: 09 September 2019

Citation: Johanna S Palve, Tiina H

Luukkaala, Minna $\mathrm{T}$ Kääriäinen.

Predictors of Metastasis and

Outcome Following True Negative

Sentinel Node Biopsy. Journal of

Surgery and Research 2 (2019):

176-184.

\begin{abstract}
Up to $30 \%$ of sentinel node-negative patients develop metastases during follow-up. Negative sentinel node biopsies (SNB) can be classified to false (FN) and true negative (TN) categories. Little attention has been paid to the characteristics and outcomes of patients who experience direct distant metastasis following TN-SNB. In this retrospective study of a melanoma database at Tampere university hospital we analyzed characteristics and outcome following metastases after TN-SNB. A total of 506 patients underwent SNB between 2006 and 2016. After review, SNBs were classified FN, TN and true positive (TP). Follow-up was performed until 30.4.2019.
\end{abstract}

Of SN-negative patients, 74 of 396 (19\%) developed recurrence, including $17(4 \%)$ local, 22 (6\%) regional lymph node (FN) and 35 (9\%) direct distant metastases (TN-D). False negative rate was $16 \%$ and negative predictive value $93.8 \%$. Locoregional recurrences occurred earlier compared to distal metastases (median of 2.14/2.93 years). Compared to patients without recurrence, thickness $\geq 2 \mathrm{~mm}$ (univariable $\mathrm{p}<0.001$ ), male gender $(\mathrm{p}=0.021$ ), nodular melanoma $(\mathrm{p}=0.001)$, ulceration $(\mathrm{p}<0.001)$ and location in upper limb region $(\mathrm{p}=0.062)$ were predictors of TN-D. The 5-year melanoma specific survival in TN-D patients did not differ significantly from TP patients $(2.36 / 2.26$ years $)$. 
TN-D is associated with nodular melanomas in upper limb region, male gender, cervical SNBs and ulcerated tumors with Breslow thickness $\geq 2 \mathrm{~mm}$. These patients should be considered at high-risk relapse and mortality. Surveillance imaging to detect distant metastases is mandatory regardless of SNB status. In future, inclusion criteria for therapy trials for high-risk SNB-negative patients might also be worth considering.

Keywords: Melanoma; Sentinel node- negative; False negative; Recurrence; Metastasis

\section{Introduction}

The standard treatment of melanoma is wide local excision of primary tumor and sentinel node biopsy (SNB) for staging purposes [1]. The utility of SNB correlates with depth of invasion of the primary tumor, and is a routine management in patients with melanoma thicker than $1 \mathrm{~mm}$ and may be considered for thin lesions with high-risk characteristics (e.g. ulceration) [2]. SN status together with Breslow thickness and ulceration have been verified as the most important prognostic factors in melanoma [3]. However, the value of SNB as a prognostic marker may be reduced by the existence of false negative results or distant recurrence after negative SNB. The probability of developing distant or local recurrence following a negative SNB during follow-up ranges from $4 \%$ to $29 \%$ [4]. Negative SNBs can be classified to false negative (FN) and true negative (TN) categories. A FN-SNB has been defined as a recurrence of melanoma in the previously biopsied lymph node basin [4]. False negative results have been reported in 2.0-18.4\% of patients [5]. Possible etiologies of FN-SNB include poor radiographic localization of $\mathrm{SN}$, failed pathologic evaluation and failure of surgical technique to identify the SN [4]. The presence of multiple SN basins and regions of the body where lymphatic drainage is known to be complex (head/neck) has the potential to contribute to an increased risk of recurrence after negative SNB [4].

True negative SNB (TN-SNB) is defined as negative SNB without regional recurrence in the previously sampled node basin [6]. It has been suggested that melanoma which recurs after negative SNB may exhibit different tumor biology. The development of distant metastases despite negative SNB may be due to local regression of primary melanoma lesions, immunologic clearance of the melanoma in the regional lymph node basin, direct hematogenous spread of the disease or the presence of melanoma leading to obstruction of lymphatic drainage [4]. The prior research has primarily attempted to identify clinical factors associated with the occurrence of FN-SNB and the impact of FN-SNB on survival outcome $[4,6,7,8]$. Patients with FN-SNB have had a worse prognosis than patients with positive SNB [5]. However, little attention has been paid to the characteristics and outcomes of patients who experience direct distant metastasis of melanoma following TNSNB. The purpose of this study was to identify clinicopathologic characteristics and outcome associated with patients with metastases after TN-SNB.

\section{Materials and Methods}

In this retrospective study, we included 506 patients who underwent successful SNB for cutaneous melanoma at the Tampere university hospital (Finland) between 2006 and 2016. Permission to access the clinical records of the melanoma patients for the study was obtained from the scientific center of Tampere University Hospital. Retrospective review of records was performed to determine following information: age, gender, primary tumor site (head/neck, trunk, upper limb, lower limb), tumor characteristics (Breslow, ulceration, subtype (superficial spreading melanoma, (SSM), nodular melanoma (NM), lentigo maligna melanoma (LMM) and other (including acral 
melanoma)) and time of diagnosis. Clinical outcomes regarding SN included the lymph node basins (cervical, axillary, inguinal, other), number of SNs removed and results of the SNB. TP-SNB was defined as any positive SNB. Negative SNBs were divided to false negative $(\mathrm{FN})$, true negative (TN) with distant metastasis (TND), TN without recurrence (TN-NoR) and negative SNB with local recurrence (LR). FN was defined as recurrence of melanoma in the previously biopsied lymph node basin. Time of the recurrence was recorded as well as time of death and reason for death (melanoma, other).

Our technique for SNB is described here briefly. All consecutive patients with a Breslow lesion $>1 \mathrm{~mm}$ or 0.75-1 $\mathrm{mm}$ with ulceration and/or mitotic activity $>1 / \mathrm{mm}^{2}$ were considered for lymphatic mapping and SNB. A preoperative lymphoscintigraphy was performed for all patients using $99 \mathrm{mTc}$ labeled human albumin colloid injected intra-dermally before the operation. Patent blue was injected intra-dermally 10 minutes before incision (until October 2013, after which it was not used). Starting in October 2013, singlephoton emission computerized tomography/computed tomography (SPECT/CT) was performed for all melanoma patients preoperatively. Intraoperative identification of the $\mathrm{SN}$ was done with a handheld gamma probe. Radioactive nodes that had count $>10 \%$ of the most radioactive node were also considered as SNs. Histopathologic analysis of SN consisted of sectioning and staining with hematoxylin and eosin. Starting in March 2012, the immunohistochemical markers S-100, Melan-A and HMB-4 were used routinely.

\subsection{Statistical analysis}

False negative rate (FNR) was calculated as the ratio between the false negatives and the total of false negative and true positives. Negative predictive value Journal of Surgery and Research
(NPV) was calculated as [Number of true negatives/(number of false negatives + number of true negatives)]. Predictive factors for SN were calculated by univariable using Mann-Whitney test, Pearson chisquare test or Fisher's exact test, and by multivariable performing logistic regression analysis. Disease-free survival (DFS) was defined as the time between the time of diagnosis and detected recurrence. Survival was calculated from the time of diagnosis to death, either from melanoma (melanoma-specific survival, MSS) or other causes (overall survival, OS). Time-to-event analyses were performed using Cox proportional hazard regression analysis. A p-value of $<0.005$ was considered significant. All analyses were performed using IBM SPSS Statistics for Windows, version 25.0 software (IBM Corp., Armonk, NY).

\section{Results}

\subsection{Overall demographics}

From 2006 to 2016, 506 patients (274 (54\%) men and $232(46 \%)$ women) underwent SNB. Follow-up was performed until 30.4.2019. The median age of patients was 68 (range 21-90) years in men and 70 (range 36-90) years in women. The median Breslow thickness was 2.0 (IQR 1.2-4.0) $\mathrm{mm}$ in men and 1.8 (IQR 1.1-3.0) $\mathrm{mm}$ in women. The most common $(66 \%, 334 / 506)$ histologic subtype was superficial spreading melanoma (SSM), followed by nodular melanoma (NM) (20\%, 102/506), lentigo maligna melanoma (LMM) $(8 \%, 42 / 506)$ and other (including acral melanoma) $(6 \%, 28 / 506)$. The most common location of melanoma was trunk $(182 / 506,36 \%)$, followed by lower limb (116/506, $23 \%)$, upper limb $(110 / 506,22 \%)$ and head/neck area (98/506, 19\%). Of 506 melanomas, 200 (40\%) were ulcerated. One hundred and ten of 506 patients (22\%) had nodal metastasis in SNB and were considered TPs. A total of 396 patients out of 506 (78\%) did not have metastasis in SNB and were considered SN-negatives. 
False negative rate was $16 \%$ and negative predictive value $93.8 \%$.

\subsection{Recurrence patterns}

The majority $(322 / 396,81 \%)$ of SN-negative patients did not have recurrence during the study period. A total of 74 out of 396 (19\%) SN-negative patients developed recurrence, including 17 (4.3\%) local (LR), 22 (5.5\%) regional lymph node (FN) and 35 (8.8\%) direct distant metastases (TN-D) (Table 1). Of SN-positive patients, 55 of $110(50 \%)$ patients developed metastases, including 39 (71\%) distant and 16 (29\%) locoregional metastases. In SNB-negative patients locoregional recurrences occurred earlier with DFS median of 2.14 years (IQR 1.09-3.50, range 0.3-4.8) compared to 2.93 (IQR 1.15-4.49, range 0.3-7.4) for distal metastases. The median DFS in TPs was 1.44 (IQR 0.82-3.08, range 0.1-4.9).

\subsection{TN-D compared with TN-NoR and TP groups}

We compared the characteristics of the TN-D patients to those of the TN-NoR and TP groups (Table 1). Compared with TN-NoR, TN-D patients were older (univariable $\mathrm{p}=0.004$, multivariable OR 1.02 (0.991.05)), more male predominant $\mathrm{p}=0.021$, OR 1.87 (0.794.49)) with Breslow thickness thicker than $2 \mathrm{~mm}$ $(\mathrm{p}<0.001$, OR $4.11(1.55-10.9)$ and ulcerated $(\mathrm{p}<0.001$, OR 1.73 (0.77-3.93). Nodular melanomas were more common in TN-D group ( $\mathrm{p}=0.001$, OR 1.97 (0.78-4.95). Upper limb region (OR 1.67 (0.55-5.09) melanomas were more common in TN-D group compared to TNNoR group. In contrast, relative to TP, TN-D patients were also more male predominant $(\mathrm{p}=0.302$, OR 1.77 (0.63-4.97) but did not have significantly increased Breslow thickness $(\mathrm{p}=0.580$, OR $1.06(0.33-3.41))$ and were less commonly ulcerated $(\mathrm{p}=0.231$, OR 0.37 (0.140.99)). Sentinel lymph node basin was more commonly in head/neck region in TN-Ds compared to both TNNoR and TP $(28 \%$ compared to $7 \%$ and $11 \%$ Journal of Surgery and Research respectively). Number of sentinel nodes removed did not differ significantly between TN-D, TN-NoR and TP groups (with median of 2.6 (range 1-6), 2.0 (1-12), 2.75 (range 0-8) respectively).

\subsection{Survival analysis}

The 5-year MSS for the entire cohort $(\mathrm{n}=506)$ was 5.23 (IQR 3.40-8.42, range 0.4-13.3) years and 78.7\%. The 5 -year MSS was similar between the TN-D and the TP group (2.36 years (IQR 1.49-3.29, range 0-5.0) compared to 2.26 years (IQR 1.49-3.29, range 0-5.0), age-adjusted HR 1.54 (95\% CI 0.84-2.81). The TN-NoR group had significantly better MSS compared with both the TP and the TN-D groups with 5-year MSS of 5.00 (IQR 3.28-5.00, range 0.4-5.0).

\section{Discussion}

Our study demonstrates that direct distant metastases after negative SNB are associated with an ulcerated tumor with Breslow thickness $\geq 2 \mathrm{~mm}$, more commonly upper limb primary tumor site, cervical SNB location, higher number of nodular melanomas and male gender. Consistent with previous publications $[4,5,9,10]$ on SNB-negative patients (including FN patients), our study revealed increasing Breslow thickness and ulceration to be predictors for distant recurrence. Of $\mathrm{SN}$-negative patients with direct distant metastases, $80 \%$ of patients had Breslow thickness $\geq 2 \mathrm{~mm}$, which has been significantly associated with relapse in melanomas in general [2]. Over half of TN-D melanomas were ulcerated compared to melanomas without recurrence, of which $26 \%$ were ulcerated. According to studies, the presence of ulceration seems to be strongly associated with hematogenous dissemination of metastasis (2), which may also explain direct distant metastases in our cases. 


\begin{tabular}{|c|c|c|c|c|c|c|c|c|c|c|c|}
\hline \multirow[b]{4}{*}{$\begin{array}{l}\text { Age }(\text { yrs })(\text { Median }(\mathrm{Md}) \pm \\
\text { range) }\end{array}$} & \multirow{4}{*}{\begin{tabular}{|l|}
$\begin{array}{l}\text { Local } \\
(\mathbf{L R})\end{array}$ \\
$(\mathbf{N}=17)$ \\
\\
$69(49-93)$
\end{tabular}} & \multirow{4}{*}{$\begin{array}{l}\text { Regional } \\
(\mathbf{F N}) \\
(\mathbf{N}=22) \\
\\
59(41-91)\end{array}$} & \multirow{4}{*}{ 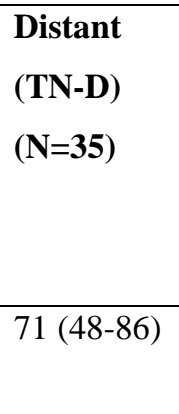 } & \multirow{4}{*}{\begin{tabular}{|l|}
$\begin{array}{l}\text { No } \\
\text { recurrence } \\
(\mathrm{TN}-N o R) \\
(\mathrm{N}=322)\end{array}$ \\
$66(18-97)$
\end{tabular}} & \multirow{4}{*}{\begin{tabular}{|l|}
$\begin{array}{l}\text { Positive } \\
\text { SNB (TP) } \\
(\mathbf{N}=110)\end{array}$ \\
$68(21-90)$ \\
\end{tabular}} & \multicolumn{3}{|c|}{$\begin{array}{l}\text { TN-D vs. TN-NoR } \\
(n=357)\end{array}$} & \multicolumn{3}{|l|}{$\begin{array}{l}\text { TN-D vs. TP } \\
(n=145)\end{array}$} \\
\hline & & & & & & \multirow{2}{*}{$\begin{array}{l}\text { Univariable } \\
\text { p-value }\end{array}$} & \multicolumn{2}{|c|}{ Multivariable } & \multirow{2}{*}{$\begin{array}{l}\text { Univariable } \\
\text { p-value }\end{array}$} & \multicolumn{2}{|c|}{ Multivariable } \\
\hline & & & & & & & OR & $(95 \% \mathrm{CI})$ & & OR & $(95 \% \mathrm{CI})$ \\
\hline & & & & & & 0.004 & 1.02 & $(0.99-1.05)$ & 0.034 & 1.04 & \begin{tabular}{|l}
$(0.997-1.09)$ \\
\end{tabular} \\
\hline \multicolumn{6}{|l|}{ Sex, n (\%) } & 0.021 & - & - & 0.302 & - & - \\
\hline Men & $4(24)$ & $13(59)$ & $25(71)$ & $164(51)$ & $68(62)$ & - & 1.87 & $(0.79-4.49)$ & - & 1.77 & $(0.63-4.97)$ \\
\hline Women & $13(76)$ & $9(41)$ & $10(29)$ & $158(49)$ & $42(38)$ & - & 1.00 & - & - & 1.00 & - \\
\hline \multicolumn{6}{|l|}{ Ulceration, n (\%) } & $<0.001$ & - & - & 0.231 & - & - \\
\hline No & \begin{tabular}{|l|}
$9(53)$ \\
\end{tabular} & $10(45)$ & $15(43)$ & $237(74)$ & $35(32)$ & - & 1.00 & - & - & 1.00 & - \\
\hline Yes & $8(47)$ & $12(55)$ & $20(57)$ & $85(26)$ & $75(68)$ & - & 1.73 & $(0.77-3.93)$ & - & 0.37 & $(0.14-0.99)$ \\
\hline $\begin{array}{l}\text { Tumor thickness }(\mathrm{mm}), \mathrm{Md} \\
\text { (IQR) }\end{array}$ & $3.5(2.5-4.0)$ & $2.7(1.9-5.1)$ & $3.5(2.0-6.5)$ & $1.5(1.1-2.4)$ & $3.0(2.0-5.0)$ & $<0.001$ & - & - & 0.580 & - & - \\
\hline $\mathrm{Br}<2 \mathrm{~mm}, \mathrm{n}(\%)$ & 1(6) & $6(27)$ & $7(20)$ & $206(64)$ & 27.(24) & - & 1.00 & - & - & 1.00 & - \\
\hline $\mathrm{Br} \geq 2 \mathrm{~mm}, \mathrm{n}(\%)$ & $16(94)$ & $16(73)$ & $28(80)$ & $116(36)$ & $83(76)$ & & 4.11 & $(1.55-10.9)$ & & 1.06 & $(0.33-3.41)$ \\
\hline \multicolumn{6}{|l|}{ Subtype, n (\%) } & 0.001 & - & - & 0.274 & - & - \\
\hline SSM & $9(53)$ & $14(64)$ & $16(46)$ & $239(74)$ & $56(51)$ & - & 1.00 & - & - & 1.00 & - \\
\hline LMM & $2(12)$ & $3(14)$ & $5(14)$ & $26(8)$ & $6(5)$ & - & 3.28 & $(0.89-12.1)$ & - & 6.32 & $(1.14-35.0)$ \\
\hline NM & $4(23)$ & $3(14)$ & $13(37)$ & $44(14)$ & $38(35)$ & - & 1.97 & $(0.78-4.95)$ & - & 1.67 & $(0.59-4.75)$ \\
\hline \multicolumn{6}{|l|}{ Tumor location, $\mathrm{n}(\%)$} & 0.062 & - & - & 0.002 & - & - \\
\hline Head and neck & $2(12)$ & $3(14)$ & $10(29)$ & $69(21)$ & $14(13)$ & - & 1.00 & - & - & 1.00 & - \\
\hline Trunk & $7(41)$ & $5(23)$ & $11(31)$ & $114(36)$ & $45(41)$ & - & 1.10 & $(0.35-3.48)$ & - & 0.38 & $(0.12-1.20)$ \\
\hline Upper limb & $2(12)$ & $5(23)$ & $10(29)$ & $73(23)$ & $20(18)$ & - & 1.67 & $(0.55-5.09)$ & - & 1.13 & $(0.31 .4 .09)$ \\
\hline Lower limb & $6(35)$ & $8(36)$ & $5(14)$ & $66(20)$ & $31(28)$ & - & 0.43 & $(0.07-2.47)$ & - & 0.12 & $(0.02-0.67)$ \\
\hline
\end{tabular}




\begin{tabular}{|l|l|l|l|l|l|l|l|l|l|l|}
\hline \multicolumn{2}{|l|}{ Exitus during follow-up, $\mathrm{n}(\%)$} & $<0.001$ & - & - & 0.008 & - & - \\
\hline of melanoma & $3(18)$ & $11(50)$ & $21(60)$ & $0(0)$ & $33(30)$ & - & & - & - & - \\
\hline of other reasons & $2(12)$ & $1(5)$ & $2(6)$ & $50(16)$ & $15(14)$ & - & & - & - & - \\
\hline
\end{tabular}

Univariable analyses were performed using Mann-Whitney test, Pearson chi-square or Fisher's exact test. In multivariable logistic regression analysis, adjusting factors (age, sex, categorized tumor thickness and tumor location) were included simultaneously into the model. Results were shown by odds ratios (OR) with 95\% confidence intervals (CI). SSM=superficial spreading melanoma, LMM=lentigo maligna melanoma, NM=nodular melanoma. Local $(\mathrm{LR})=$ local recurrence, Regional $(\mathrm{FN})=$ regional lymph node basin recurrence (false negative cases), Distant $(\mathrm{TN}-\mathrm{D})=$ direct distant metastasis after true negative sentinel node biopsy, No Recurrence $(\mathrm{TN}-\mathrm{NoR})=$ melanoma cases with no recurrence during follow-up, Positive SNB (TP)=melanoma cases with positive sentinel node biopsy (true positive cases).

Table 1: Clinicopathologic characteristics and outcome for patients after sentinel node biopsies (SNB). 
In our study, the location of melanoma in head/neck region was not statistically associated with distant recurrences after true negative $\mathrm{SNB}$ in multivariate analysis, which has been found in previous studies with false negative cases $[4,5,6,10]$. It has been suggested that the increased rate of recurrences despite of negative SNB (false negative) in the head and neck region may be rated to technical factors, including the complex drainage patterns of that region [6]. Unexpected or aberrant lymph drainage patterns are expected in head and neck melanomas more than melanomas located on upper and lower extremity [10]. This supports more routine use of SPECT-CT in this population [7]. We studied occurrence of direct metastases after true negative SNB and this could explain this difference compared to other studies. We have used SPECT-CT from October 2013 to all melanoma patients routinely preoperatively. Before that, conventional 2-dimensional lymphoscintigraphy was performed. The impact of this change in SNB results was not evaluated in this study.

In our study, however, the higher number of melanoma in the upper limb region was associated with direct distant metastases. The upper extremity primary has been reported earlier [11] to predict $\mathrm{SN}+$ status but has not been connected to SN-negative melanomas. In our study, $18 \%$ of TP patients compared to $28 \%$ of TN-Ds had melanoma in upper limb area. The most common location of $\mathrm{SN}$ in upper limb region is axilla but the lymphatic drainage from upper parts of upper limb might also go to cervical area. In our study, sentinel lymph node basin was more commonly in head/neck region in TN-Ds compared to both TN-NoRs and TPs.

On the other hand, the question of reason for recurrence might not be the location of SNs. Several other explanations have been suggested. The possibility of immunologic clearance of the melanoma in the regional lymph node basin prior to SNB may be responsible for the development of distant metastases despite negative SNB [9]. It has been also suggested that especially ulceration may reflect a distinct subtype of melanoma with a higher vascular density and greater local host response [12]. Similarly, changes in the tumor microenvironment in ulcerated melanoma may interact differently with the immune system [5]. Metastatic melanoma leading to obstruction of lymphatic drainage and direct hematogenous spread of the disease have been proposed as a possible etiology of metastatic disease following a negative SNB [4].

An association between NM and melanoma recurrence has been described in SN-negative patients [10]. In our study $37 \%$ of TN-D patients had NM compared to $14 \%$ of patients with no recurrence. It has been hypothesized that the aggression of NM may be because of decreased recognition by immune system, as represented by a lower levels of tumor-infiltrating lymphocytes compared to SSMs [13]. It is possible that differences in the immunogenicity of the primary melanoma are relevant for recurrence after negative SNB [5] This difference in the levels of tumor-infiltrating lymphocytes, however, appeared to be prominent in melanomas thinner than $2 \mathrm{~mm}$, while the difference was no longer significant in melanomas thicker than $2 \mathrm{~mm}$ [13].

The relationship of gender to TN-D is curious and currently unexplained. While it is well known that male gender is an adverse prognostic factor in melanoma, it is not clear why this would be related to the accuracy of lymphatic mapping [6] On the other hand, immunologic differences in response to tumor may contribute toward sex differences in melanoma outcome. Men may show less antitumor surveillance, resulting in reduced immune recognition of nodular melanoma. For example, lower levels of tumor-infiltrating lymphocytes in NMs compared to SSMs have been observed only in men [13]. 
The other major issue examined in this study is the impact of TN-D on outcome. The prior research has primarily attempted to identify clinical factors associated with the occurrence of FN-SNB and the impact of FN-SNB on survival outcome $[4,6,7,8]$. According to other studies, patients with FN-SNB have worse prognosis than patients with positive SNB at least in longer than 5 years follow-up [5, 6]. Little attention has, however, been paid to the characteristics and outcomes of patients who experience direct distant metastasis of melanoma following TN-SNB. In our study, when analyzed with 5 years follow-up, there was no significant difference between TPs and TN-Ds. It should be, however, noted that most recurrences in melanoma occurs within 5 years and $80 \%$ of patients who experience recurrences after the first 5 years follow-up are SNB-negative. Thus, the late recurrences in patients with negative SNB may influence the 10year predictive role of SNB status in the Cox regression analysis [2]. It is important, in future, to analyze 10 years MSS also in our study cohort.

This study has the limitations inherent to any retrospective study with potential confounding variables that we have not accounted for. The current study is not able to provide a causative explanation for the direct distant metastases after negative SNB, although the identification of various patient and tumor factors associated with metastases would argue for at least a partial role for tumor biology. A prospective study with a larger sample of SNB-negative patients with subsequent recurrence would be helpful to refine the analysis of risk factors in TN-D patients.

It would be impractical and not cost efficient to propose that all patients with negative SNB should undergo increased surveillance. We undertook this study to examine the factors and clinical implications of their occurrence that are associated with TN-D. The current Journal of Surgery and Research study identifies high- risk groups for TN-D events for whom counseling and change in clinical management and surveillance would be appropriate. While the value of earlier detection of metastatic disease has been at best unclear historically, with the recent advent of effective novel therapeutic agents for melanoma, changes might be possible. In future, inclusion criteria for therapy trials for high-risk SNB-negative patients might also be worth considering.

\section{Conclusion}

In this study, the association of TN-D with nodular melanomas more commonly in upper limb tumor site, cervical SNB location, male gender and ulcerated tumors with Breslow thickness $\geq 2 \mathrm{~mm}$ was described. These patients should be considered at high-risk relapse and mortality and we therefore recommend stricter follow-up to these patients regardless of SNB status. In future, inclusion criteria for therapy trials for high-risk SNB-negative patients might also be worth considering.

\section{Funding}

There was no funding for this study.

\section{Conflict of Interest}

All authors declare that they have no conflict of interest.

\section{References}

1. Borgogni L, Bellucci $F$, Urso $C$, et al. Enhancing the prognostic role of melanoma sentinel lymph nodes through microscopic tumour burden characterization: clinical usefulness in patients who do not undergo complete lymph node dissection. Melanoma Research 29 (2019):163-171.

2. Portinari M, Baldini G, Guidoboni M, et al. The long-term prognostic impact of sentinel lymph node biopsy in patients with primary cutaneous melanoma: a prospective study with 
10-year follow-up. Ann Surg Treat Res 95 (2018): 286-296.

3. Belgrano V, Katsarelias D, Mattsson J, et al. Sentinel node for malignant melanoma: An observational study of a consecutive single center experience. Eur J Surg Oncol 45 (2019): 225-230.

4. Hodges M, Jones E, Jones T, et al. Analysis of melanoma recurrence following a negative sentinel lymph node biopsy. Melanoma Manag 2 (2015): 285-294.

5. Persa O, Knuever J, Mauch C. Risk factors for recurrence of malignant melanoma in patients with negative sentinel lymph node biopsy. J Dtch Dermatol Ges Jun 20 (2019).

6. Lee D, Huynh K, Teng A, et al. Predictors and survival impact of false-negative sentinel nodes in melanoma. Ann Surg Oncol 23 (2016): 1012-1018.

7. Sinnamon A, Neuwirth M, Bartlett E, et al. Predictors of false negative lymph node biopsy in trunk and extremity melanoma. J Surg oncol 116 (2017): 848-855.

8. Erdmann M, Uslu U, Göhl J, et al. Riski factors for regional and systemic metastases in patients with sentinel lymph node-negative melanoma. Anticancer Res 38 (2018): 65716577.

9. Yee V, Thopson J, McKinnon G, et al. Outcome in 846 cutaneous melanoma patients from a single center after a negative sentinel node biopsy. Ann Surg Oncol 12 (2005): 1-11.

10. Faut M, Wevers $\mathrm{K}$, van Ginkel $\mathrm{R}$, et al. Nodular histologic subtype and ulceration are tumor factors associated with high risk of recurrence in sentinel node-negative melanoma patients. Ann Surg Oncol 24 (2017): 142-149.

11. Ellis $\mathrm{M}$, Weerasinghe $\mathrm{R}$, Corless $\mathrm{C}$, et al. Sentinel lymph node staging of cutaneous melanoma: predictors and outcomes. Am J Surg 199 (2010): 663-668.

12. De Moll E, Fu Y, Perkins S, et al. Immune biomarkers are more accurate in prediction of survival in ulcerated than in non-ulcerated primary melanomas. Cancer Immunol and Immunother 64 (2015): 1193-1203.

13. Lin R, Wang $\mathrm{T}$, Joyce $\mathrm{C}$, et al. Decreased tumor-infiltrating lymphocytes in nodular melanomas compared with matched superficial spreading melanomas. Melanoma Research 26 (2016): 524-527. 